\title{
Beyond probiotics the Postbiotics
}

\begin{abstract}
The term postbiotic was introduced to describe a product containing dead microorganisms and their metabolites, Soluble factors (products or metabolic by-products),Secreted by live bacteria, (OR) Released after bacterial lysis, such as enzymes, peptides, teichoic acids, peptidoglycan-derived muropeptides, polysaccharides, cell surface proteins, and organic acids.
\end{abstract}

Keywords: metabolite, probiotics, SCFA, imunity
Volume 10 Issue 6 - 2019

\author{
Aziz Koleilat \\ Department of Pediatrics, Beirut Arab University, Lebanon \\ Correspondence: Aziz Koleilat, Department of Pediatrics, \\ Beirut Arab University, Vice General Secretary of Pan Arab \\ Society Pediatric Gastroenterology Hepatology And Nutrition, \\ Makassed University General Hospital, Beirut, Lebanon, Tel \\ 009613231717; 0096 I 130038, Email drkoleilat@hotmail.com, \\ pediatric@makassedhospital.org \\ Received: November 15, 2019 | Published: December 30, \\ 2019
}

Abbreviations: SCFAs, short-chain fatty acids; LPS, lipopolysaccharide; ZO-1, Zonula occludens-1

\section{Mini Review}

Postbiotics are defined as "any factor resulting from the metabolic activity of a probiotic or any released molecule capable of conferring beneficial effects to the host in a direct or indirect way. ${ }^{1}$ The term postbiotic was introduced to describe a product containing dead microorganisms and their metabolites such as soluble factors secreted by live bacteria or released after bacterial lysis, including enzymes, peptides, teichoic acids, cell surface proteins, polysaccharides, and organic acids. ${ }^{2}$ Postbiotics are also known as: "Para probiotics", "Non-viable probiotics", "Inactivated probiotics", "Ghost probiotics", or "Metabiotic, biogenic". All are inactivated microbial cells, which, when administered in sufficient amounts, confer benefits to consumers. ${ }^{1,3}$

Postbiotics may contribute, to the improvement of host health by improving specific physiological functions. Although the exact mechanisms have not been entirely elucidated; yet, postbiotics have clear chemical structure, safety dose parameters and long shelf life. They contain several signaling molecules which may have anti-inflammatory, immunomodulatory, anti-obesogenic, anti-hypertensive, hypo-cholesterolemic, anti-proliferative, and antioxidant activities. ${ }^{2}$

Postbiotics are non-pathogenic, non-toxic and resistant to hydrolysis by mammalian enzymes. ${ }^{4}$ Dead probiotics have been shown to modulate the immune system. Compounds of the cell wall might boost the immunological system. Probiotics increase adhesion to intestinal cells which further results in inhibition of pathogens. Postbiotics have various epigenetic processes such as DNA methylation, phosphorylation, biotinylating, histone acetylation and RNA interference. These have been involved in the epigenetic control of the host cell responses. ${ }^{5,6}$ The modifications affect immunomodulation, competitive exclusion, and regulating epithelial cell barrier function. These biochemical modifications exert their beneficial role in the prevention of various deadly diseases including cancers, IBD, auto-immune disorders, and life style disorders. ${ }^{7}$
There are various forms of postbiotics such as peptidoglycans, peptide molecules, organic acids or short chain fatty acids, exopolysaccharides, and bacteriocins (Table 1). ${ }^{8}$ These could elicit several biological responses since they have absorption, metabolism and distribution abilities indicating a high capacity to signal different tissues and organs in the host. ${ }^{1,2,9,10}$ Most of postbiotics active components are produced during fermentation including shortchain fatty acids (SCFAs), peptides, enzymes, cell surface proteins, polysaccharides, and vitamins. ${ }^{2}$ SCFAs are a source of energy for cells and help regulate energy homeostasis. They possess anti-oxidative, anti-carcinogenic and anti-inflammatory properties. They play an important role in the immune system. ${ }^{11}$

Postbiotics stimulate healthy gut microbiota and support immune function through the gut. ${ }^{12}$ They maintain gut health. The bioactive probiotic-derived components have a similar protective role on intestinal barrier function as that of live probiotics. ${ }^{13-15}$ These bioactive components enhance intestinal mucin expression, prevent lipopolysaccharide (LPS), or tumor necrosis factor $\alpha$ (TNF- $\alpha$ ) to induce intestinal barrier injury, down regulate intestinal mucin (MUC2), and enhance Zonula occludens-1 (ZO-1) (Tight Junction). ${ }^{16}$

They also protect against disruption of the intestinal integrity. They boost immune function by improving the ability to fight infection such as increasing the antibody response to pathogens and influencing gut barrier function and intestinal immunity. Postbiotics enhance barrier function against species like Saccharomyces boulardii, and improve angiogenesis in vitro and in vivo in epithelial cells by activation of $\alpha 2 \beta 1$ integrin collagen eceptors. ${ }^{17-23}$ Probiotic species of Bifidobacterium breve, Bifidobacterium lactis, Bifidobacterium infantis, Bacteroides fragilis, Lactobacillus, Escherichia coli and Faecalibacterium prausnitzii have similar properties for postbiotics. ${ }^{9}$

If we can summarize the postbiotics effects, postbiotics consist of microbial metabolites and microbial component. The microbial metabolites include enzymes (GPx, SOP, NADH-peroxidase), protein /peptides (glutathione), polysaccharides and organic acids, as well as lipids (short chain fatty acids). The microbial components are lipoteichoic acids, peptidoglycan, teichicacids, cell surface proteins 
and polysaccharides. These have an effect in the lumen on the gut epithelium and on the lamina propria. In the lumen they have local effect on immunomodulation, anti-inflammatory and anti- microbial properties, while on the gut epithelium they have effect on the tight junction and on mucin activity. Whereby on the Lumina propria they have systemic effect such as anti-oxidant, anti-hypertensive, antiobesogenic and anti-proliferative effect to all body organs. ${ }^{2}$

Table I Postbiotics active components and their natural sources

\begin{tabular}{|c|c|c|}
\hline $\begin{array}{l}\text { Bioactive compounds } \\
\text { Postbiotics Probiotics }\end{array}$ & Natural sources & References \\
\hline - $\quad$ Bacteriocins & Lactobacillus plantarum I-UL4 & Ooi MF'9 \\
\hline - Heat-killed LGG & Lactobacillus rhamnosus & Islam SU20 \\
\hline - Soluble mediator & Lactobacillus paracasei & Tsilingiri $\mathrm{K}^{21}$ \\
\hline - Butyrate & Faecalibacterium prausnitzii & Giorgetti GM17 \\
\hline - Polyphosphate & Lactobacillus brevis & Zagato $E^{21}$ \\
\hline - Exopolysaccharide & Lactobacillus pentosus & Sornplang $\mathrm{P}^{22}$ \\
\hline - $\quad$ Short-chain fatty acids & Lactobacillus gasser & Tiptiri-Kourpeti $\mathrm{A}^{23}$ \\
\hline
\end{tabular}

\section{Conclusion}

Potential beneficial health effects of dead microorganisms deserve to be taken into consideration than living organisms. Although living microorganisms are needed to restore or influence the intestinal microbiome composition, particles of the microorganisms and/or their metabolites may also be sufficient to induce immunological effects. The discovery of postbiotics leads to the progressive drive towards research and progression in this field. They have been found to possess capability of immunomodulation, pathogen exclusion and maintenance of gastro-intestinal integrity. Their epigenetic effects at molecular level will serve as potential molecules for abrogating various diseases, in prevention and maybe treatment.

\section{Acknowledgments}

To Miss Lubna Sino Research department of Makassed University General Hospital for editing and corrections.

\section{Conflicts of interest}

None.

\section{Funding}

None.

\section{References}

1. Tsilingiri K, Rescigno M. Postbiotics: what else? Benef Microbes. 2012;4(1):101e7.

2. Aguilar-Toalá JE, García-Varela R, García HS et al. Postbiotics: An evolving term within the functional foods field. Trends Food Sci Technol. 2018;75:105-114.

3. Taverniti V, Guglielmetti S. The immunomodulatory properties of probiotic microorganisms beyond their viability (ghost probiotics: proposal of paraprobiotic concept). Genes Nutr. 2011;6(3):261-274.

4. Kerry RG, Patra JK, Gouda S, et al. Benefaction of probiotics for human health: A review. J food Drug Anal. 2018;26(3):927-939.
5. Paul B, Barnes S, Demark-Wahnefried W, et al. Influences of diet and the gut microbiome on epigenetic modulation in cancer and other diseases. Clin Epigenetics. 2015;7:112.

6. Shenderov BA. Gut indigenous microbiota and epigenetics. Microb Ecol Health Dis. 2012;23:17195.

7. Sheflin AM, Whitney AK, Weir TL. Cancer-promoting effects of microbial dysbiosis. Curr Oncol Rep. 2014;16(10):406.

8. Malashree L, Vishwanath Angadi K, Shivalkar Y, Prabha, V. "Postbiotics"-One Step Ahead of Probiotics. Int J Curr Microbiol App Sci. 2019;8:2049-2053.

9. Cicenia A, Scirocco M, Carabotti L, Pallotta M, Marignani C. Severi C. Postbiotic activities of lactobacilli-derived factors. J Clin Gastroenterol. 2014;48(Suppl 1):S18-S22.

10. Suez J, Elinav E. The path towards microbiome-based metabolite treatment. Nat Microbiol. 2017;2:17075.

11. Thorburn AN, Macia L, Mackay CR. Diet, metabolites, and "western life style" inflammatory diseases. Immunity. 2014;40(6):833-842.

12. Patel R, Denning PW. Therapeutic Use of Prebiotics, Probiotics, and Postbiotics to Prevent Necrotizing Enterocolitis. What is the Current Evidence? Clin Perinatol. 2013;40(1):11-25.

13. Lebeer S, Bron PA, Marco ML, et al. Identification of probiotic effector molecules: present state and future perspectives. Curr Opin Biotechnol. 2018;49:217-223.

14. Konishi H, Fujiya M, Tanaka H, et al. Probiotic-derived ferrichrome inhibits colon cancer progression via $\mathrm{JNK}$-mediated apoptosis. Nat Commun. 2016;7:12365.

15. Sanchez B, Urdaci MC, Margolles A. Extracellular proteins secreted by probiotic bacteria as mediators of effects that promote mucosa-bacteria interactions. Microbiology. 2010;156(Pt 11):3232-3242.

16. Gao J, Li Y, Wan Y, et al. A novel postbiotic from Lactobacillus rhamnosus GG with a beneficial effect on intestinal barrier function. Front Microbiol. 2019;10:477. 
17. Giorgetti GM, Brandimarte G, Fabiocchi F, et al. Interactions between innate immunity, microbiota, and probiotics. J Immunol Res. 2015;2015.

18. Singhal B, Vishwakarma V, Singh A. Metabiotics: The Functional Metabolic Signatures of Probiotics: Current State-of-Art and Future Research Priorities-Metabiotics: Probiotics Effector Molecules. Advances in Bioscience and Biotechnology. 2018;9(4):720-726.

19. Ooi MF, Mazlan N, Foo HL, et al. Effects of carbon and nitrogen sources on bacteriocin inhibitory activity of postbiotic metabolites produced by Lactobacillus plantarum I-UL4. Malays J Microbiol. 2015;11:176e84.

20. Islam SU. Clinical uses of probiotics. Medicine (Baltimore). 2016;95(5):1e5.
21. Zagato E, Mileti E, Massimiliano L, et al. Lactobacillus paracasei CBA L74 metabolic products and fermented milk for infant formula have anti-inflammatory activity on dendritic cells in vitro and protective effects against colitis and an enteric pathogenin vivo. PLoS One. 2014;9(2):1e14.

22. Sornplang P, Piyadeatsoontorn S. Probiotic isolates from unconventional sources: a review. J Anim Sci Tech. 2016;58:1e11.

23. Tiptiri-Kourpeti A, Spyridopoulou K, Santarmaki V, et al. Lactobacillus casei exerts anti-proliferative effects accompanied by apoptotic cell death and up-regulation of TRAIL in colon carcinoma cells. PLoS One. 2016;11(2):1e20. 